\title{
Actualidad en manejo de abcesos prostáticos: a propósito de un caso
}

\author{
Serrano Frago P, Allepuz Losa C, Fantova Alonso A, Marco Valdenebro A, Allué López M, \\ Rioja Sanz LA.
}

Servicio de Urología. Hospital Universiario Miguel Server. Zaragoza.

Actas Urol Esp. 2006;30(7):720-722

\section{RESUMEN}

ACTUALIDAD EN MANEJO DE ABCESOS PROSTÁTICOS: A PROPÓSITO DE UN CASO

La existencia de abscesos prostáticos como mala evolución de una prostatitis aguda no es muy frecuente dado al correcto manejo de las infecciones urinarias mediante antibioterapia adecuada, a pesar de ello existen situaciones de inmunosupresión que pueden favorecer su aparición.

Es nuestro propósito revisar la actuación en estas situaciones a raíz de un caso que mediante un drenaje percutáneo mínimamente invasivo consiguió completa mejoría.

Palabras clave: Absceso prostático. Tratamiento. Derivación percutánea.

\section{ABSTRACT}

THE PRESENT TIME IN HANDLING OF PROSTATE ABSCESSES: WITH REGARD TO A CASE

The existence of prostate abscess as bad evolution of an acute protatitis is not very frequent due to the correct handling of the urinary infections by means of suitable antibiotics, in spite of the presence of inmunosupresion situations that can favor their appearance. It is our intention to review the performance in these situations because of a case that by means of minimal invasive therapy by transperineal percutaneous puncture obtained complete improvement.

Keywords: Prostate abscess. Treatment. Percutaneous drainage.

$\mathrm{L}$ a presencia de abscesos prostáticos es poco frecuente y en la mayoría de las ocasiones que aparece está asociada a una prostatitis sobre todo en pacientes diabéticos o inmunodeprimidos. Se diagnostica fundamentalmente mediante ecografia transrectal o tomografía computarizada y su tratamiento consiste en antibioterapia y drenaje del mismo de manera transperineal, transrectal o transuretral.

\section{CASO CLINICO}

Varón de 38 años que acude a urgencias de nuestro hospital por empeoramiento de la clínica miccional y fiebre de hasta $38^{\circ} \mathrm{C}$. Llevaba en tratamiento antibiótico con levofloxacino 5 días, por un diagnóstico mediante sedimento patológico y cortejo clínico de disuria y polaquiuria de prostatitis aguda, sin obtener urocultivo.
Acude al hospital por mala evolución clínica con imposibilidad para la micción, dolor testicular bilateral y perineal y empeoramiento del estado general.

A la exploración, se evidencia una temperatura de $38,6^{\circ} \mathrm{C}$, constantes vitales mantenidas y presencia de una tumefacción en la zona perineal con enrojecimiento y calor local (Fig. 1). No se pudo realizar tacto rectal debido al intenso dolor que le produjo el intento.

En las pruebas complementarias realizadas, se evidencia una leucocitosis: 18.000 leuc/mm3, piocitos en el sedimento de orina estando el resto de parámetros dentro de la normalidad.

La prueba de imagen diagnostica llevada a cabo es una ecografía abdominal y transperineal, en las que se evidencia presencia de orina en vejiga y un absceso multiloculado prostático. 


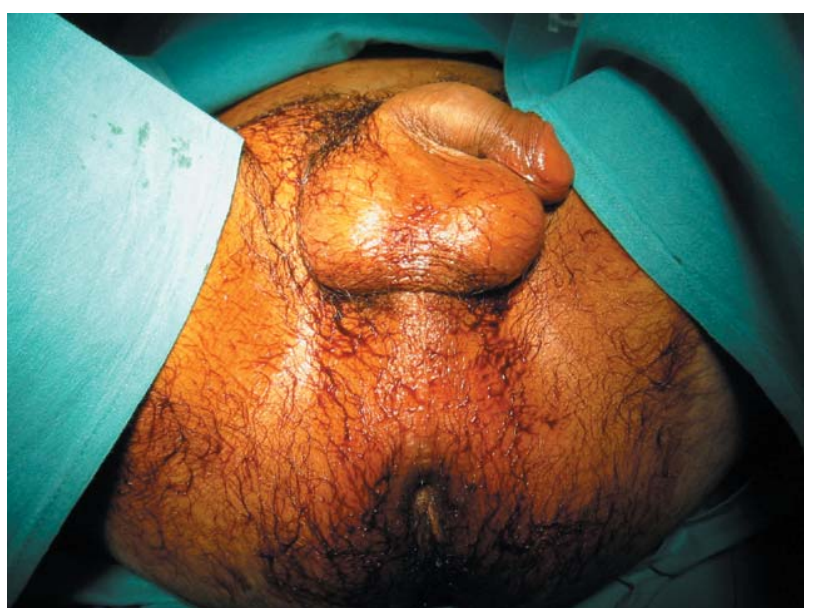

FIGURA 1: tumefacción en zona perineal.

Sin contraindicación general previa y bajo sedación se realiza de urgencia un drenaje percutáneo del absceso y colocación de un catéter suprapúbico. Se realiza una punción transperineal ecodirigida obteniendo contenido purulento y se deja "pig-tail" de 7 French como drenaje (Figs. 2 y 3 ).

Los cultivos del contenido purulento y de la orina salieron negativos, probablemente falseados por el antibiótico administrado previamente.

La evolución del paciente fue favorable, disminuyendo el débito del "pig-tail" durante 24 horas y retirándose posteriormente. Encontrándose afebril y con micción uretral normal fue dado de alta comprobándose ecográficamente al mes la normalidad de la zona prostática (Fig, 4).

\section{DISCUSION}

Los abscesos prostáticos son una complicación conocida y bien descrita de las prostatitis aguda pero poco frecuentes. Se sabe que su aparición está favorecida por la presencia de una inmunosupresión, sonda permanente, diabetes y en ancianos ${ }^{1}$.
Se debe sospechar la presencia de un absceso prostático ante una clínica urinaria de origen infeccioso con mala evolución a pesar de tratamiento antibiótico pudiéndose evidenciar masa flemonosa prostatica ${ }^{2}$.

La etiología más frecuente, al igual que las infecciones urinarias en el varón, se deben a Gram negativos, en su mayoría a Escherichia $\mathrm{Coli}^{3}$. El método mas fiable para el diagnostico de absceso prostático es la ecografía.

El tratamiento se lleva a cabo mediante una terapia combinada de antibioterapia intravenosa y drenaje del absceso. Para drenar el absceso se han descrito varias técnicas como drenaje mediante resección trasuretral, drenaje trasrectal y transperineal ecográfico, y quirúrgico abierto, siendo este último abandonado por la mínima invasión del resto de opciones ${ }^{4}$. La realización de esta modalidad percutánea de drenaje tiene la ventaja de poder realizarse con anestesia local,

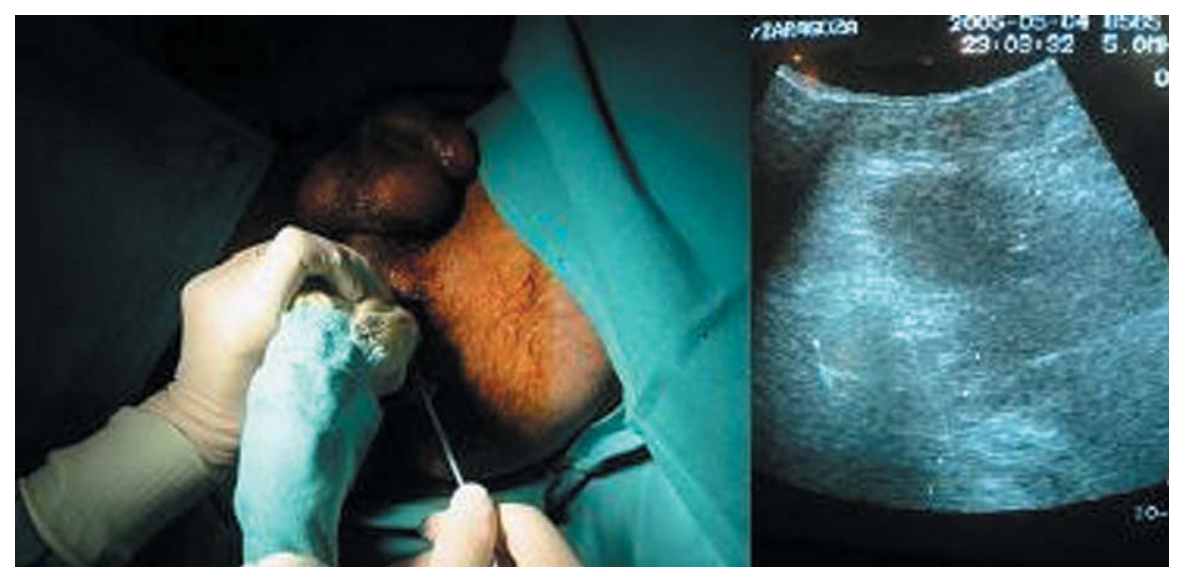

FIGURA 2: drenaje percutáneo ecodirigido.

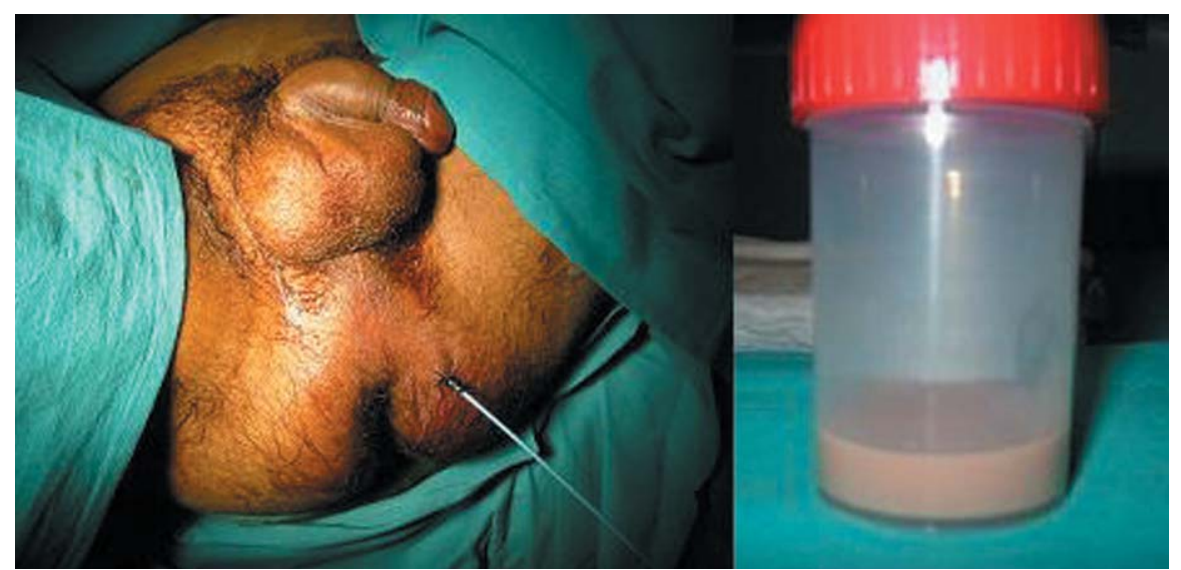

FIGURA 3: drenaje con "pig-tail" y contenido purulento extraido. 


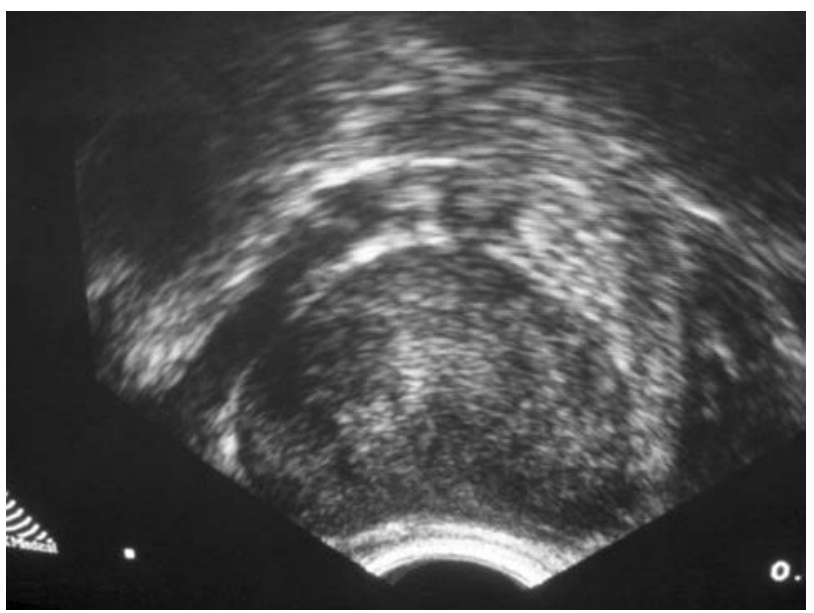

FIGURA 4: ecografia normal de control al mes.

que en situaciones de máxima urgencia y gravedad por la sepsis, sobre todo en pacientes añosos, puede ser de gran utilidad. Las variantes técnicas descritas además varían en cuanto a realizar aspiración del contenido o dejar además un drenaje $^{5}$. En las series reflejadas en la literatura se demuestra una correcta evolución de los casos sin requerir drenajes quirúrgicos y sin presentar recurrencias ${ }^{6}$.

Las complicaciones por un diagnostico tardío o mal drenaje de un absceso prostático incluyen desde la ruptura espontánea a uretra, vejiga, periné o recto hasta prostatitis crónica, infertilidad o sepsis ${ }^{7}$.
Por todo lo dicho, nosotros realizamos y aconsejamos realizar el drenaje de los abcesos de origen prostático por vía transperineal percutánea.

\section{REFERENCIAS}

1. Duarte Ojeda JM, García Luzón A, Carero VM, Vázquez S, Acalora L, Pasas J, et al. Tubérculos prostática absceso in a patient with AIDS. Actas Urol Esp. 1995;19(8):655-61.

2. Franco A, Menéndez V, Luque MP, et al. Absceso prostático: diagnóstico y tratamiento. Actas Urol. Esp. 1996;20:189 192 .

3. M. Bosquet Sanz, V. Gimeno Argente, J.L. Palmero Martí, M.A. Bonillo García, J.V. Salom Fuster, J.F. Jiménez Cruz. Absceso prostático: revisión de la literatura y presentación de un caso. Actas Urol Esp 2005;29(1):100-104.

4. Collado A, Palou J, García-Penit J, et al. Ultrasound-guided needle aspiration in prostatic abscess. Urology 1999; 53:548-552.

5. Herard A, Brandt B, Colin J, Drancourt E, Youinou Y, Lardennois B. Prostatic abscesses: what treatment to propose? Prog Urol. 1999;9(4):767-771.

6. Chou YH, Tiu CM, Liu JY, Chen JD, Chiou HJ, Chiou SY, et al. Prostatic abscess: transrectal color Doppler ultrasonic diagnosis and minimally invasive therapeutic management. Ultrasound Med Biol. 2004;30(6):719-724.

7. Granados EA, Riley G, Salvador J, Vincente J. Prostatic abscess: diagnosis and treatment. J Urol. 1992 Jul; 148(1):80-82.

Dra. P. Serrano Frago

Servicio de Urología

Hospital Universitario Miguel Servet

C/ Paseo Isabel la católica 1-3

50009 Zaragoza

E-mail: pserranof@comz.org

(Trabajo recibido el 6 de septiembre 2005) 